\title{
Aerobic exercise training improves mitochondrial biogenesis and oxidative status in obese mice with nonalcoholic fatty liver disease: a non-randomized experimental study
}

Matheus Santos de Sousa Fernandes ( $\nabla$ theusfernandes10@hotmail.com ) Universidade de Sao Paulo https://orcid.org/0000-0002-1066-9176

Lucas de Lucena Simões e Silva

Universidade de Sao Paulo

Márcia Saldanha Kubrusly

Universidade de Sao Paulo

Talitta Ricarlly Lopes de Arruda Lima

Universidade Federal de Pernambuco

Cynthia Rodrigues Muller

Universidade de Sao Paulo

Anna Laura Viacava Américo

Universidade de Sao Paulo

Mariana Pinheiro Fernandes

Universidade Federal de Pernambuco

Bruno Cogliati

Universidade de Sao Paulo

Jose Tadeu Stefano

Universidade de Sao Paulo

Claudia J Lagranha

Universidade de Sao Paulo

Fabiana Sant'Anna Evangelista

Universidade de Sao Paulo

Claudia P Oliveira

Universidade de Sao Paulo

Research article

Keywords: liver disease, mitochondria, oxidative stress, leptin deficiency

Posted Date: October 28th, 2019 
DOI: https://doi.org/10.21203/rs.2.16512/v1

License: (c) (1) This work is licensed under a Creative Commons Attribution 4.0 International License. Read Full License 


\section{Abstract}

Non-alcoholic fatty liver disease (NAFLD) is one of the most common forms of liver disease. Lifestyle modifications, such as a reduction in body weight (BW) and aerobic exercise training (AET), are effective treatments for NAFLD. The aim of the present study was to evaluate the effect of AET on hepatic oxidative metabolism in ob/ob mice. Male ob/ob mice were separated into two groups: the sedentary group (S), $n=7$, and the trained group $(T), n=7$. The T mice were submitted to an 8-week protocol of AET at $60 \%$ of the maximum velocity achieved in the running test. Before AET, no difference was observed in running capacity between the groups $(S=10.4 \pm 0.7 \mathrm{~min}$ vs. $T=13 \pm 0.47 \mathrm{~min})$. However, after $A E T$, the running capacity was increased in the T group $(12.8 \pm 0.87 \mathrm{~min})$ compared to the $S$ group $(7.2 \pm 0.63 \mathrm{~min})$. Skeletal muscle in the T group $(26.91 \pm 1.12 \mathrm{U} / \mathrm{mg}$ of protein) showed higher citrate synthase activity compared with the $S$ group $(19.28 \pm 0.88 \mathrm{U} / \mathrm{mg}$ of protein) $(p=0.004)$. BW and food consumption were significantly reduced in the T group compared to the $S$ group ( $p=0.008$ and $p=0.001$, respectively). The analysis of hepatic gene expression showed an increase in PGC-1a levels $(p=0.002)$ and a reduction in CPT-1a levels $(p=0.03)$. The levels of TBARs and carbonyls, as well as SOD, CAT and GST, were not different between the groups. In the nonenzymatic antioxidant system, we found that the T group had higher sulfhydryl ( $p=0.02), G S H(p=0.001)$ and GSH/GSSG $(p=0.02)$ activity. The activity of the metabolic enzymes citrate synthase $(p=0.004)$ and $\beta-H A D(p=0.01)$ was also increased in the T group. Besides improve in metabolism, no differences were observed in the histological analyses. In conclusion, our data demonstrate that AET improves BW control, mitochondrial functionality and oxidative metabolism in ob/ob mice.

\section{Background}

Non-alcoholic fatty liver disease (NAFLD) is characterized by the accumulation of intrahepatic lipids, reaching an absolute level of $5 \%$ of the total hepatic content $[1,2]$. It is associated with several etiologic factors and metabolic comorbidities, such as insulin resistance (IR), cardiovascular diseases (CVD), and type 2 diabetes (T2DM), and is responsible for the progression of clinical staging for non-alcoholic steatohepatitis (NASH), liver cirrhosis and hepatocellular carcinoma (HCC)[3]. Currently, NAFLD is one of the most common causes of liver diseases and affects approximately $25-30 \%$ of people around the world. In Western society, its prevalence reaches alarming levels of $20-30 \%$ in overweight adults and $90 \%$ in obese adults [3]. In addition, the process of industrialization, an imbalance in the intake of macronutrients and sedentarism contribute to the development and progression of this pathology [4].

One of the important factors that contributes to the progression of the NAFLD spectrum and the progression to advanced disease is the production of excess reactive oxygen species (ROS) (mainly superoxide anions and peroxides), which are responsible for the direct stimulation of the chronic inflammatory process, the deregulation of oxidative metabolism, and the promotion of fibrogenesis through the activation of hepatic stellate cells followed by the increased expression of type I collagen in the hepatic parenchyma $[5,6]$. Among the substances that are produced is hydrogen peroxide $\left(\mathrm{H}_{2} \mathrm{O}_{2}\right)$; in the intracellular environment, this substance causes the activation of macrophages, which in turn express 
pro-inflammatory cytokines. In addition, metabolic imbalance provides functional overload in the mitochondria, which ultimately causes mitophagy, the process of mitochondrial degradation, and the subsequent autolysis of this organelle [7]. These processes are regulated by the incremental expression of peroxisome proliferator-activated receptor gamma coactivator 1-alpha (PGC1-) and its signaling pathway and have important repercussions for energy metabolism and glucose metabolism control, mainly in the stabilization of energy deficits in the liver and in the whole organism that are triggered by overweight and obese phenotypes and NAFLD [8].

Regarding pharmacological treatment, there is no FDA-approved direct therapy for NAFLD, although some medications are used to control etiological factors such as hyperinsulinemia and IR [3, 9]. Nevertheless, changes in lifestyle, including adherence to regular aerobic exercise training (AET) and better dietary patterns, are recommended as the first line of treatment for NAFLD $[10,11]$. It has been demonstrated that AET exerts an important role in the control of NAFLD physiopathology by reducing body weight and oxidative stress, improving oxidative metabolism, and reducing steatosis, inflammation and the levels of fibrosis biomarkers, such as cytokeratin 18 [12-14]. In addition, AET at a moderate-vigorous intensity increases cardiorespiratory capacity and prevents the development and progression of NAFLD [15]. Moreover, studies have shown that AET may decrease the detrimental effect of ROS production in addition to increasing the rate of muscle contraction, beta-oxidation and mitochondrial biogenesis [1618]

Although the repercussions of AET in NAFLD are already known $[18,19]$, the effects of AET on oxidative metabolism (oxidative stress, redox state and metabolic activity) in the liver are not completely understood. Thus, the aim of the present study was to evaluate the effect of AET in the liver of $o b / o b$ mice. $o b / o b$ mice were used because the development of obesity associated with leptin deficiency can be mimicked with great reproducibility in this strain. The development of obesity associated with leptin deficiency produces hyperphagic behavior, insulin resistance and reduced movement capacity, which combine to initiate the development of NAFLD. Our hypothesis is that AET improves oxidative metabolism, decreases oxidative stress and improves histological parameters in the liver of obese mice with nonalcoholic fatty liver disease.

\section{Methods}

\section{- Animals}

Male 6-week-old $o b / o b$ mice with leptin deficiency were obtained by a genetically modified lineage with detection by genotyping method, where the recessive homozygous genotype represented the present leptin deficit obese phenotype. [from the Laboratório de Gastroenterologia Clínica e Experimental (LIM07)], matched for body weight, were separated randomly into two groups: sedentary $(S, n=7)$ and trained $(T, n=7)$. The mice were housed in a temperature-controlled environment $\left(22 \pm 2{ }^{\circ} \mathrm{C}\right)$ with a $12-\mathrm{h} \mathrm{light} / 12-\mathrm{h}$ dark cycle and free access to tap water and food (Nuvilab - Nuvital Nutrientes S/A, Brazil). The procedures were performed according to the recommendations of the Brazilian College for Animal 
Experimentation and were approved by the Ethics Committee of the School of Medicine of University of Sao Paulo (Number 040/17).

\section{- Running Test}

The test was performed before, in the fourth and eighth weeks of AET using a progressive method without inclination described by Ferreira et al. [20]. The protocol started with a speed of $0.4 \mathrm{~km} / \mathrm{h}$ and has been increased by $0.2 \mathrm{~km} / \mathrm{h}$ every three minutes until mice exhaustion.

\section{- Aerobic Exercise Training}

T mice were trained during the dark cycle on a motorized treadmill (KT 10200, Brazil) for $1 \mathrm{~h} /$ day at $60 \%$ of maximal velocity, five times per week for eight weeks. The AET intensity progressively increased; it started at $0.3 \mathrm{~km} / \mathrm{h}$ and was adjusted after the running capacity test performed in the fourth week. S mice were placed on the treadmill for $10 \mathrm{~min}$ twice weekly at $0.2 \mathrm{~km} / \mathrm{h}$ to minimize treadmill stress.

\section{- Body Weight and Food Intake}

Body weight was measured weekly at the same time of day using a digital balance (Gehaka, Model BK4001, Brazil), and 24-h food intake was determined weekly throughout the study. The mice were housed in cages containing 3-4 mice [21].

\section{- Euthanasia}

Forty-eight hours after the end of the last training session, the mice were anesthetized with an intraperitoneal ketamine hydrochloride $(0.5 \mathrm{~mL} / \mathrm{kg})$, and exsanguination was performed and consequent tissue removal. The liver was harvested, weighed and processed according to the experiments described.

\section{- Gene Expression}

After liver and skeletal muscle tissue $(50 \mathrm{mg})$ pulverization at liquid nitrogen temperatures, total RNA was prepared using Trizol® (Invitrogen Life Technologies, Carlsbad, CA, USA) according to the manufacturer's recommendations [8]. Total RNA was dissolved in RNase-free water, and the RNA concentration was determined by spectrophotometry. RNA purification was determined based on a 260/280 nm ratio $>1.8$. 
Samples were kept at $-80^{\circ} \mathrm{C}$ until processing by reverse transcription quantitative polymerase chain reaction (RT-qPCR) analysis.

After extracting the total RNA, the expression levels of multiple genes in the liver were measured. The genes that were measured and the primers used to measure them are as follows: sterol regulatory element-binding protein 1 (SREBP1) (5'GCG CTA CCG GTC TTC TAT CA; 3' GGA TGT AGT CGA TGG CCT $T G$ ); peroxisome proliferator-activated receptor alpha (PPAR-a) (5` ATG CCA GTA CTG CCG TTT TC; 3' TTG CCC AGA GAT TTG AGG TC); carnitine palmitoyl transferase I (CPT-1 a) (5' TGC CTC TAT GTG GTG TCC AA; 3' TCA AAC AGT TCC ACC TGC TG); peroxisome proliferator-activated receptor gamma coactivator 1-alpha (PGC-1a) (5'CTA CAG ACA CCG CAC ACA TCGC; 3’ GGA TGT AGT CGA TGG CCT TG); and endogenous control gene $\beta$-actin (5' TGT TAC CAA CTG GGA CGA CA; 3' GGG GTG TTG AAG GTC TCA AA). The levels were analyzed in the liver with the polymerase Rotor gene 3000 (Corbett Research, Sydney, Australia) using the Superscript ${ }^{\text {TM }}$ III Platinum ${ }^{\circledR}$ One-Step Quantitative RT-PCR System (Invitro-gen Life Technologies, Carlsbad, USA) according to the instructions provided by the manufacturer. Reactions lacking reverse transcriptase were also run to generate controls for the assessment of genomic DNA contamination. Fluorescence changes were monitored after each cycle $\left(72{ }^{\circ} \mathrm{C}\right.$, ramping to $99^{\circ} \mathrm{C}$ at 0.2 ${ }^{\circ} \mathrm{C} / \mathrm{sec}$, with continuous fluorescence readings), and melting curve analyses were performed at the end of the cycles to verify the PCR product identity. After the experiment was performed, the relative amount of each intergroup gene was calculated by the ${ }^{\mathrm{DD}} \mathrm{Ct}$ coefficient, as provided by the device software [22].

\section{- Histopathological analysis}

Fragments of liver tissues were fixed in formaldehyde (4\%) in saline and processed for hematoxylineosin (HE) staining. The histological features of NAFLD activity included macrovesicular steatosis (0-3), microvesicular steatosis (0-3), hypertrophy of the hepatocytes (0-3) and inflammatory focus (0-3). These features were assessed using a semiquantitative score designed specifically for rodent models [23].

\section{Enzyme activity}

Citrate synthase is the first enzyme in the Krebs cycle and is very important for the catalysis and condensation of acetyl CoA with oxaloacetate for the formation of citrate, the first product of the Krebs cycle. Moreover, this enzyme is an indicator of trainability, as described by Le Page et al. (2009) [18]. Briefly, the reaction was carried out in a mixture containing Tris- $\mathrm{HCl}(\mathrm{pH}=8.2)$, magnesium chloride $(\mathrm{MgCl})$, ethylenediamine-tetra-acetic acid (EDTA), 0.2-5.5 dithiobis (2-nitrobenzoic acid) $(\mathrm{E}=13.6 \mu \mathrm{mol} /$ $(\mathrm{mL} . \mathrm{cm}), 3$ acetyl CoA, 5 oxaloacetate and $0.3 \mathrm{mg} / \mathrm{mL}$ of homogenized hepatic and skeletal muscle tissue. The enzymatic activity was evaluated by measuring the change in the absorbance rate at $412 \mathrm{~nm}$ for $3 \mathrm{~min}$ at a temperature of $25^{\circ} \mathrm{C}$. Citrate levels are expressed as $\mathrm{U} / \mathrm{mg}$ of protein [24].

For the measurement of $\beta-H A D$ activity in liver, the liver was homogenized in a solution containing the following (in millimolar concentrations): $20 \mathrm{Tris}-\mathrm{HCl}\left(\mathrm{pH} 7.4\right.$ at $\left.4{ }^{\circ} \mathrm{C}\right), 50 \mathrm{NaCl}, 50 \mathrm{NaF}, 5$ 
Sodium pyrophosphate, 0.25 sucrose, and dithiothreitol, with protease inhibitor cocktail (Sigma) and phosphatase inhibitor cocktail (Sigma) [26]. After homogenization, the protein contents of the homogenates were determined by the Bradford protein assay. For $\beta$-HAD activity, $15 \mu \mathrm{g}$ of protein was incubated in a reaction mixture containing the following (in millimolar concentrations): 50 imidazole ( $\mathrm{pH}$ 7.4), $0.15 \mathrm{NADH}$, and 0.1 acetoacetyl-CoA (omitted for the control). $\beta-H A D$ activity was determined at 340 $\mathrm{nm}$ by measuring the consumption of NADH $\left(\varepsilon 6.22 \mu \mathrm{mol} \cdot \mathrm{ml}^{-1} \cdot \mathrm{cm}^{-1}\right)$ over $5 \mathrm{~min}$ (in 30-s intervals), $\beta$-HAD levels are expressed as $\mathrm{U} / \mathrm{mg}$ of protein. The procedures that were used were described previously by lto et al. (2010)[25].

\section{- Oxidative Stress}

In the present study, lipid peroxidation was also evaluated through substances reactive to thiobarbituric dosage. In this assay $300 \mu \mathrm{g}$ of protein were mixed to $30 \%(\mathrm{w} / \mathrm{v})$ trichloroacetic acid (TCA) and $3 \mathrm{mM}$ TRIS buffer ( $\mathrm{pH} \mathrm{7.4)} \mathrm{in} \mathrm{equal} \mathrm{volumes} \mathrm{and} \mathrm{stirred.} \mathrm{This} \mathrm{mixture} \mathrm{was} \mathrm{centrifuged} \mathrm{at} 2,500 \mathrm{~g}$ for $10 \mathrm{~min}$, the supernatant was mixed with $0.73 \%$ thiobarbituric acid and boiled at $100^{\circ} \mathrm{C}$ for $15 \mathrm{~min}$. The pink pigment yielded was measured spectrophotometrically at $535 \mathrm{~nm}$ at room temperature. The results were expressed as $\mu \mathrm{mol}$ of $\mathrm{MDA} / \mathrm{mg}$ protein) $[26,27]$.

The protein oxidation was evaluated as described by Levine et al. (1990). Liver samples with $300 \mathrm{mg}$ of protein, 30\% (w/v) TCA was added to the sample and then centrifuged for $15 \mathrm{~min}$ at $664 \mathrm{~g}$. The pellet was resuspended in $10 \mathrm{mM}$ 2,4- dinitrophenylhydrazine (DNPH) and immediately incubated in a dark room for $1 \mathrm{~h}$ with shaking every $15 \mathrm{~min}$. The samples were washed and centrifuged three times in ethyl acetate buffer and at the end of the procedures the pellet was resuspended in $6 \mathrm{M}$ guanidine hydrochloride incubated for $30 \mathrm{~min}$ at $37 \mathrm{o} 163 \mathrm{C}$ and the absorbance read at $370 \mathrm{~nm}$. The results were expressed as $\mu \mathrm{M} / \mathrm{mg}$ protein [28].

\section{- Antioxidant defense}

Superoxide dismutase (SOD) activity was determined 166 in agreement with Misra and Fridovich (1972) [29]. In this way, $300 \mu \mathrm{g}$ of protein was used with addition of $100 \mathrm{mM}$ of carbonate buffer with $5 \mathrm{mM}$ EDTA $(\mathrm{pH}$ 10.2). The reaction was initiated with the addition of $150 \mathrm{mM}$ epinephrine and the SOD activity was determined by the inhibition of epinephrine auto-oxidation at $30^{\circ} \mathrm{C}$. The decrease in absorbance was monitored for $2 \mathrm{~min}$ at $480 \mathrm{~nm}$ and the results express in $\mathrm{U} / \mathrm{mg}$ protein.

Catalase activity assay has been previously described Aebi (1984) [30]. Liver homogenate (300 $\mu \mathrm{g}$ of the protein) was used, with the addition of $50 \mathrm{mM}$ of the phosphate buffer $(\mathrm{pH} 7.0)$ and $0.3 \mathrm{M}$ of hydrogen 
peroxide and its oxidation. All enzymatic kinetics was monitored at $240 \mathrm{~nm}$ for $3 \mathrm{~min}$ at $20^{\circ} \mathrm{C}$, and the results expressed as $\mathrm{U} / \mathrm{mg}$ protein [27].

The activity of GST was previously described by Habig et al (1974) [31] that evaluated as follows: $200 \mu \mathrm{g}$ protein was added to $100 \mathrm{mM}$ phosphate buffer (pH 6.5) containing 1 mM EDTA. For this solution, $1 \mathrm{mM}$ reduced glutathione and $1 \mathrm{mM}$ 1- chloro-4,4-dinitrobenzene (CDNB) were added so that the reaction could start. The absorbance standard for this component was monitored at $340 \mathrm{~nm}$ for $1 \mathrm{~min}$ to detect the formation of 2,4-dinitrophenol-S-glutathione (DNP-SG). One enzyme unit conjugates $10 \mathrm{nmol}$ of CDNB with GSH per minute. The results were expressed as $\mathrm{U} / \mathrm{mg}$ protein.

To measure the REDOX state, we measured both reduced and oxidized glutathione levels. The levels of reduced glutathione (GSH) were evaluated by adding $100 \mathrm{mM}$ phosphate buffer (pH 8.0) with $5 \mathrm{mM}$ EDTA to the samples $(0.300 \mathrm{mg}$ protein), followed by a period of $15 \mathrm{~min}$ incubation with 0 phthalaldehyde (OPT) $(1 \mu \mathrm{m})$ at RT. Fluorescence intensity was measured at $350 \mathrm{~nm}$ (excitation) and 420 $\mathrm{nm}$ (emission) and compared with a standard GSH curve (0.5-100 $\mu \mathrm{M})$. The oxidized glutathione (GSSG) levels were evaluated by incubation of samples with $40 \mathrm{mM}$ Nethylmaleimide for a period of $30 \mathrm{~min}$ in RT followed by addition of $100 \mathrm{mM} \mathrm{NaOH}$ buffer. Afterwards, the same steps of the GSH assay were followed 196 to determine the GSSG levels. The REDOX state was determined by the ratio of GSH/GSSH [32].

The measurement of total thiol groups consisted of a cold extraction buffer ( $50 \mathrm{mM}$ Tris base, $\mathrm{pH} 7.4 ; 1$ mM EDTA; 2 mM PMSF, 10 mM sodium orthovanadate) added to the samples, followed by incubation with $10 \mathrm{mM}$ 5,5'-dithiobis (2 nitrobenzoic acid) (DTNB) at RT under a dark cover for a period of 30 min. The samples were measured at $412 \mathrm{~nm}$ as described by Ellman [33].

\section{- Statistical Analysis}

Data are reported as the mean \pm SEM. Differences between the two groups were analyzed using Student's t-test, except for body weight evolution, which was analyzed using one-way ANOVA for repeated measures. The Bonferroni post hoc test was used to determine differences between the means when a significant change was determined by ANOVA. For the analysis of histopathological factors, a semiquantitative analysis with statistical treatment using chi-square $\left(\chi^{2}\right)$ was used for categorical variables. A $p$ value of less than 0.05 was statistically significant, and Prism V6 was used.

\section{Results}

Before AET, no difference was observed in the running capacity between the groups ( $\mathrm{S}=10.4 \pm 0.7 \mathrm{~min} v \mathrm{~s}$. $\mathrm{T}=13 \pm 0.47 \mathrm{~min})$. However, after $\mathrm{AET}$, the running capacity was increased in the $\mathrm{T}$ group $(12.8 \pm 0.87$ $\mathrm{min})$ compared to the $\mathrm{S}$ group $(7.2 \pm 0.63 \mathrm{~min})$. We analyzed the activity of citrate synthase in skeletal muscle as an indicator of AET efficiency. Our data demonstrated that the T group $(26.91 \pm 1.12 \mathrm{U} / \mathrm{mg}$ of 
protein) exhibited higher citrate synthase activity compared to that exhibited by the $S$ mice $(19.28 \pm 0.88$ $\mathrm{U} / \mathrm{mg}$ of protein).

In the present study, the body weight of the animals in both groups at the beginning of AET was evaluated, and no statistically significant differences were observed (Figure 1A). During the experimental protocol, the T mice exhibited lower body weight beginning in the fourth week of AET compared to that exhibited by the $S$ group. Body weight gain was lower in the T group compared to the S group (Figure 1B; $p=0.037$ ). The $T$ mice consumed less food than the $S$ mice (Figure $1 C ; p=0.001$ ).

The T mice exhibited higher citrate synthase activity and $\beta-H A D$ enzyme levels in the liver when compared to those in the liver of $S$ mice ( $p=0.01$ and 0.003 , respectively) (Figures $2 A$ and $2 B$ ). No differences were found in the mRNA levels of SREBP1 and PPAR-a ( $p=0.309$ and 0.615 , respectively) (Figures 2C and 2D). In contrast, we observed decreased mRNA expression of CPT-1a $(p=0.03)$ (Figure 2E) and a significant increase in PGC-1 a mRNA expression ( $p=0.002)$ in the T mice (Figure 2F).

Regarding oxidative stress, no difference was observed in lipid and protein peroxidation between the groups ( $p=0.6167$ and 0.0887 ) (Figures $3 A$ and $3 B$ ). The activity of antioxidant enzymes (SOD, CAT and GST $)$ in the livers of the T and S mice were also not significantly different $(p=0.06,0.11$ and 0.08 , respectively) (Figures 3C, 3D and 3E). However, the T group showed higher levels of nonenzymatic defense [GSH levels, redox state (GSH/GSSG ratio), and the amount of sulfhydryl groups] compared to those in the $S$ group $(p=0.001, p=0.02$ and $p=0.02)$ (Figures $4 A, 4 B$ and $4 C$, respectively).

Histological analysis (Table 1) showed that the mice from both groups presented similar characteristics of all parameters in histology. The $\chi^{2}$ test did not reveal significant differences between the groups $(p=$ 0.965).

\section{Discussion}

Our study was designed to test the hypothesis that AET improves oxidative metabolism, decreases oxidative stress and improves histological parameters in the liver of obese mice with nonalcoholic fatty liver disease. Our results revealed that AET was responsible for an improvement in body weight control, a decrease in hyperphagic behavior, and an improvement in $\beta$-oxidation and oxidative status in association with an increase in the expression of genes related to mitochondrial biogenesis in the liver of $o b / o b$ mice with NAFLD.

In the present study, variables related to AET were analyzed to determine trainability, that is, the adaptability of responses related to physical exercise. From this perspective, the T mice showed higher mean values of running capacity (measured as the length of time spent running in min) compared to those of the $\mathrm{S}$ mice. This result demonstrates that AET can provide greater physical fitness in these mice, indicating higher levels of cardiorespiratory adaptation or aerobic capacity after AET at a moderate intensity. This result was also confirmed by higher citrate synthase activity in skeletal muscle of the T 
group and corroborates the findings of Evangelista et al., which demonstrated that eight weeks of AET were able to increase duration time assessed by the running capacity test [21].

A recent study published by Loombaet al. [11] showed that weight loss at different levels can generate a number of benefits for the hepatic and metabolic health of patients with NAFLD and that 52 weeks of physical activity can cause $10 \%$ body weight loss, leading to the reversal of NASH (a more severe form of NAFLD) in $90 \%$ of patients and the regression of liver failure by $81 \%$. Similarly, in a study carried out by our group, T mice showed better control of body weight evolution; however, the differences between the groups began in the third week of AET [21]. These data were also observed in our study, thus demonstrating the efficacy and reproducibility of AET in body weight control.

Overweight, obese and NAFLD patients commonly experience an increase in the incidence of cardiometabolic diseases, physical inactivity, hormonal dysregulation, T2DM and other conditions[12, 34]. In the liver, excess fat is directly responsible for IR, pro-inflammatory processes, ROS, energy dysfunction, fibrogenesis and cellular apoptosis $[35,36]$.

The literature shows that the generation of NAFLD is regulated by innumerable factors (including the abnormal accumulation of intrahepatic lipids, insulin resistance, and a reduction in $\beta$-oxidation capacity), but none of these factors are as important as mitochondrial dysfunction. Thyfault et al. (2009) [37] reported that such energetic dysregulations are mediated by different levels of PGC-1 gene expression. This coactivator regulates the activation and deactivation of a signaling pathway for mitochondrial biogenesis which involves CPT-1, PPAR-transcription factor A, mitochondrial (TFAM), nuclear respiratory factor-1 and 2 (NFR-1 and 2). Another recent study showed that physically inactive mice have lower levels of PGC-1 gene expression and that this has repercussions for the establishment of an obesogenic phenotype, histological damage, oxidative stress and NAFLD $[18,19,38]$.

In our study, when we evaluated PGC-1 gene expression in the liver of $o b / o b$ mice, the T group demonstrated a significant increase compared to the levels in the $S$ group. In addition, we also evaluated the levels of PPAR-; despite a trend toward higher mean values in the T mice, no significant differences were observed. However, surprisingly, in contrast to our first study, we found a significantly lower level of CPT-1 intrahepatic gene expression in the T mice than in the $S$ mice. An explanation of this result may relate to the physiological structure of these obese animals, which are exposed to constant metabolic insults arising from leptin deficiency, an important factor in the regulation of the expression of genes linked to lipogenesis and lipolysis. We hypothesize that a reduction in the expression of leptin, an acylCoA transporter, may influence the mitochondrial $\beta$-oxidation capacity or be related to the lack of differences in SREBP1 expression we observed between the groups. Consistent with our findings, Gonçalves et al. [9] showed that increases in the level of PGC-1 gene expression are a key factor in the regulation of lipid metabolism and body weight and generate benefits for important features of NAFLD.

In an earlier study, Evangelista et al. (2015) [21] found a higher level of intrahepatic CPT-1 gene expression in $o b / o b$ mice who underwent the same protocol of AET used in the current study. In addition, these mice had a better metabolic profile as measured by indirect calorimetry but did not show 
improvement in semiquantitative histological analyses or express significant levels of SREBP1. In our study, we chose to use the same experimental model; however, we decided to start the AET protocol 6 weeks after weaning. We hypothesize that the early onset of the AET intervention can improve metabolic function and pathophysiological features in these animals. We found significant differences in BW from the fourth week of AET intervention. In addition, we found no significant difference in the expression of SREBP1 in the liver, which was also observed in the previous study, and a significant increase in PGC-1a gene expression, which may generate greater mitochondrial biogenesis.

Sun et al. (2010) [39] demonstrated that 4 weeks of AET at a high intensity and for a short duration was able to increase the production of TBARs and ROS in the rat liver, whereas rats that practiced and received supplements of b-complex vitamins and creatine exhibited greater activity of mitochondrial complexes I, IV and V. This demonstrates that the effect of AET on pro-oxidant compounds and antioxidant defense should be evaluated chronically and in different intensity-duration patterns.

In the present study, there was no difference in antioxidant defense by SOD, CAT or GST in the T group. However, regular AET increases SOD, CAT and GST activity in the liver and skeletal muscle. In our study, we used genetically modified isogenic mice with leptin deficiencies and a different metabolic structure; these mice should be studied more deeply as these characteristics may have caused deregulation of the enzymatic complex. Furthermore, the duration of AET intervention may not have been long enough to promote these adaptations in the antioxidant enzymes. Moreover, reducing oxidative stress is a fundamental method for improving not only the progression of NAFLD but also obesity and IR in physically inactivity patients $[40,41]$.

Due to the results we found related to enzymatic defense, we decided to analyze some molecules related to nonenzymatic defense, such as reduced and oxidized glutathione levels and the total amount of thiol groups. Our data showed that the $T$ animals presented significantly higher levels of all the components of nonenzymatic defense compared to those exhibited by the $S$ mice. The increase in the redox levels in response to AET may be effective in reducing the intracellular amount of peroxide and hydroxyl peroxide produced and thus impact the decrease in the proinflammatory response, fibrosis and cellular apoptosis commonly found in NAFLD/NASH [32, 42]. These results are of paramount importance in the spectrum of NAFLD since the activation of the action-dependent pathway of the redox state complex guarantees the liver's protective effect against the production of bioactive compounds related to oxidative stress [42].

In addition, we performed an analysis of $\beta-H A D$ and hepatic citrate synthase activity in these animals since these elements are closely linked to mitochondrial function and activation. The $T$ animals demonstrated a significant increase in both metabolic enzymes compared to that in the S group, this result confirms the efficiency of the AET on metabolic parameters associated to mitochondrial bioenergetic providing better hepatic energetic functionality.

- $\circ$ Limitations 
As limitations in the present study, we can mention the insufficient number of weeks in which the mice underwent AET. In addition, the absence of dietary control and lipogenesis aggressiveness of the leptindeficient animal model may be associated with the absence of histopathological results.

\section{Conclusion}

Therefore, our data demonstrate that AET improves body weight control, hyperphagic behavior, mitochondrial biogenesis and oxidative status.

\section{Declarations}

\section{Abbreviations}

AET: Aerobic exercise training, NAFLD: Nonalcoholic fatty liver disease, SED: Sedentary Group, T: Trained Group; CAT: Catalase, SOD: Superoxide Dismutase, GST: Glutathione S Transferase; GSH: Reduced glutathione levels, GSSG: Oxidated glutathione levels, TBARS: Thiobarbituric acid reactive species, T2DM: Type 2 diabetes mellitus, ROS: Reactive oxygen species;

\section{Ethics approval and consent to participate}

The procedures were performed according to the recommendations of the Brazilian College for Animal Experimentation and were approved by the Ethics Committee of the School of Medicine of University of Sao Paulo (Number 040/17).

\section{Consent for publication}

Not applicable.

\section{Availability of data and materials}

The data used to support the findings of this study are available from the corresponding author upon request.

\section{Competing interests}

The authors declare that they have no competing interests.

\section{Funding}

Financial support provided by Coordenação de Aperfeiçoamento de Pessoal de Nível Superior (CAPES) and FACEPE (Grant number: APQ-0164-4.05/15).

\section{Contributions}


MSSF, CPM, LLSS, SJT and FSE conceived the study idea and design. MSSF, LLSS and FSE formulated the aerobic exercise training intervention. MSSF, CPM, LLSS, SJT and FSE conducted animal model selection and care. MSSF, CPM, LLSS, SJT, CM, ALAV and FSE conducted interventions. MSSF, LLSS and MSK performed DNA extraction and RT-PCR analysis, MSSF, LLSS, TRLAdL, MFP performed the Oxidative Status analysis and BC performed histological liver analysis. MSSF, CPM, LLSS, SJT and FSE wrote the manuscript with review, editing and final approval from all authors.

\section{Acknowledgements}

The authors are thankful for financial support provided by Coordenação de Aperfeiçoamento de Pessoal de Nível Superior (CAPES) and FACEPE.

\section{References}

1.Musso, G., et al., Dietary habits and their relations to insulin resistance and postprandial lipemia in nonalcoholic steatohepatitis. Hepatology, 2003. 37(4): p. 909-16.

2.Erlich, A. T., et al., Function of specialized regulatory proteins and signaling pathways in exerciseinduced muscle mitochondrial biogenesis. Integrative medicine research, 2016. 5(3): p. 187-197.

3.Begriche, K., et al., Mitochondrial dysfunction in NASH: causes, consequences and possible means to prevent it. Mitochondrion, 2006. 6(1): p. 1-28.

4.Perfield, J. W., 2nd, et al., Altered hepatic lipid metabolism contributes to nonalcoholic fatty liver disease in leptin-deficient Ob/Ob mice. J Obes, 2013. 2013: p. 296537.

5.Molero, J. C., et al., Casitas b-lineage lymphoma-deficient mice are protected against high-fat dietinduced obesity and insulin resistance. Diabetes, 2006. 55(3): p. 708-715.

6.Rani, V., et al., Oxidative stress and metabolic disorders: Pathogenesis and therapeutic strategies. Life Sci, 2016. 148: p. 183-93.

7.Georgoulis, M., et al., Blood redox status is associated with the likelihood of nonalcoholic fatty liver disease irrespectively of diet's total antioxidant capacity. Nutr Res, 2015. 35(1): p. 41-8.

8. Halliwell, B. and J. M. Gutteridge, Free radicals in biology and medicine. 2015: Oxford University Press, USA.

9.Gonçalves, I. O., et al., Exercise as a therapeutic tool to prevent mitochondrial degeneration in nonalcoholic steatohepatitis. European journal of clinical investigation, 2013. 43(11): p. 1184-1194.

10.Magkos, F., Exercise and fat accumulation in the human liver. Curr Opin Lipidol, 2010. 21(6): p. 50717. 
11.Loomba, R. and H. Cortez-Pinto, Exercise and improvement of NAFLD: Practical recommendations. J Hepatol, 2015. 63(1): p. 10-2.

12.Rinella, M. E. and A. J. Sanyal, Management of NAFLD: a stage-based approach. Nat Rev Gastroenterol Hepatol, 2016. 13(4): p. 196-205.

13.Sen, C. K., Oxidants and antioxidants in exercise. J Appl Physiol (1985), 1995. 79(3): p. 675-86.

14.Sen, C. K., M. Atalay, and O. Hanninen, Exercise-induced oxidative stress: glutathione supplementation and deficiency. J Appl Physiol (1985), 1994. 77(5): p. 2177-87.

15.Linden, M. A., et al., Treating NAFLD in OLETF rats with vigorous-intensity interval exercise training. Med Sci Sports Exerc, 2015. 47(3): p. 556-67.

16.Rayyan, Y. M. and R. F. Tayyem, Non-alcoholic fatty liver disease and associated dietary and lifestyle risk factors. Diabetes \& Metabolic Syndrome: Clinical Research \& Reviews, 2018. 12(4): p. 569-575.

17.Mantena, S. K., et al., Mitochondrial dysfunction and oxidative stress in the pathogenesis of alcoholand obesity-induced fatty liver diseases. Free Radic Biol Med, 2008. 44(7): p. 1259-72.

18.Koves, T. R., et al., Peroxisome proliferator-activated receptor-gamma co-activator 1alpha-mediated metabolic remodeling of skeletal myocytes mimics exercise training and reverses lipid-induced mitochondrial inefficiency. J Biol Chem, 2005. 280(39): p. 33588-98.

19.Grattagliano, I., et al., Oxidative stress-induced risk factors associated with the metabolic syndrome: a unifying hypothesis. J Nutr Biochem, 2008. 19(8): p. 491-504.

20.Ferreira, J. C., et al., Maximal lactate steady state in running mice: effect of exercise training. Clin Exp Pharmacol Physiol, 2007. 34(8): p. 760-5.

21. Evangelista, F. S., et al., Physical training improves body weight and energy balance but does not protect against hepatic steatosis in obese mice. Int J Clin Exp Med, 2015. 8(7): p. 10911-9.

22.Rao, X., et al., An improvement of the $2^{\wedge}(-$ delta delta CT) method for quantitative real-time polymerase chain reaction data analysis. Biostat Bioinforma Biomath, 2013. 3(3): p. 71-85.

23.Liang, W., et al., Establishment of a general NAFLD scoring system for rodent models and comparison to human liver pathology. PLoS One, 2014. 9(12): p. e115922.

24.Alp, P. R., E. A. Newsholme, and V. A. Zammit, Activities of citrate synthase and NAD+-linked and NADP+-linked isocitrate dehydrogenase in muscle from vertebrates and invertebrates. Biochem J, 1976. 154(3): p. 689-700.

25.Ito, M., et al., High levels of fatty acids increase contractile function of neonatal rabbit hearts during reperfusion following ischemia. Am J Physiol Heart Circ Physiol, 2010. 298(5): p. H1426-37. 
26.Buege, J. A. and S. D. Aust, Microsomal lipid peroxidation. Methods Enzymol, 1978. 52: p. 302-10.

27.Nascimento, L., et al., The effect of maternal low-protein diet on the heart of adult offspring: role of mitochondria and oxidative stress. Appl Physiol Nutr Metab, 2014. 39(8): p. 880-7.

28.Reznick, A. Z. and L. Packer, Oxidative damage to proteins: spectrophotometric method for carbonyl assay. Methods Enzymol, 1994. 233: p. 357-63.

29.Misra, H. P. and I. Fridovich, The role of superoxide anion in the autoxidation of epinephrine and a simple assay for superoxide dismutase. J Biol Chem, 1972. 247(10): p. 3170-5.

30.Aebi, H., Catalase in vitro. Methods Enzymol, 1984. 105: p. 121-6.

31.Habig, W. H., M. J. Pabst, and W. B. Jakoby, Glutathione S-transferases. The first enzymatic step in mercapturic acid formation. J Biol Chem, 1974. 249(22): p. 7130-9.

32.Hissin, P. J. and R. Hilf, A fluorometric method for determination of oxidized and reduced glutathione in tissues. Analytical biochemistry, 1976. 74(1): p. 214-226.

33.Ellman, G. L., Tissue sulfhydryl groups. Arch Biochem Biophys, 1959. 82(1): p. 70-7.

34.Jeppesen, J. and B. Kiens, Regulation and limitations to fatty acid oxidation during exercise. J Physiol, 2012. 590(5): p. 1059-68.

35.Milagro, F. I., et al., Dietary factors, epigenetic modifications and obesity outcomes: progresses and perspectives. Mol Aspects Med, 2013. 34(4): p. 782-812.

36.Serviddio, G., et al., Mitochondrial involvement in non-alcoholic steatohepatitis. Mol Aspects Med, 2008. 29(1-2): p. 22-35.

37.Thyfault, J. P., et al., Rats selectively bred for low aerobic capacity have reduced hepatic mitochondrial oxidative capacity and susceptibility to hepatic steatosis and injury. J Physiol, 2009. 587(Pt 8): p. 180516.

38.Rui, L., Energy metabolism in the liver. Compr Physiol 4: 177-197. 2014.

39.Sun, L., et al., Endurance exercise causes mitochondrial and oxidative stress in rat liver: effects of a combination of mitochondrial targeting nutrients. Life sciences, 2010. 86(1-2): p. 39-44.

40.Morris, E. M., et al., Mitochondria and redox signaling in steatohepatitis. Antioxid Redox Signal, 2011. 15(2): p. 485-504.

41.Linden, M. A., et al., Treating NAFLD in OLETF rats with vigorous-intensity interval exercise training. Medicine and science in sports and exercise, 2015. 47(3): p. 556. 
42.Shin, S.-K., et al., Catalase and nonalcoholic fatty liver disease. Pflügers Archiv-European Journal of Physiology, 2018. 470(12): p. 1721-1737.

\section{Tables}

Table. 1. Semiquantitative analysis of histopathological parameters in the liver of $o b / o b$ mice after eight weeks of AET. S $(n=4)$ and T $(n=4)$.

\begin{tabular}{|c|c|c|c|c|c|c|c|c|c|}
\hline \multirow{2}{*}{$\begin{array}{l}\text { Histopathological } \\
\text { characteristics }\end{array}$} & \multicolumn{4}{|c|}{ ID/Sedentary* } & \multicolumn{5}{|c|}{ ID/Trained } \\
\hline & 1 & 2 & 3 & 4 & 5 & 6 & 7 & 8 & $p\left(x^{2}\right)$ \\
\hline $\begin{array}{l}\text { Macrovesicular steatosis } \\
\qquad(0-3)\end{array}$ & 0 & 2 & 1 & 2 & 1 & 2 & 2 & 0 & 0,965 \\
\hline $\begin{array}{l}\text { Microvesicular steatosis } \\
\qquad(0-3)\end{array}$ & 0 & 3 & 2 & 3 & 2 & 3 & 2 & 3 & \\
\hline $\begin{array}{l}\text { Hypertrophy of hepatocytes } \\
\qquad(0-3)\end{array}$ & 0 & 2 & 2 & 2 & 1 & 2 & 1 & 2 & \\
\hline $\begin{array}{l}\text { Inflammatory focus } \\
\qquad(0-3)\end{array}$ & 0 & 2 & 3 & 3 & 2 & 2 & 3 & 0 & \\
\hline
\end{tabular}

* Identification carried out by microchip; $\chi^{2}$ : chi-square test

\section{Figures}


A)

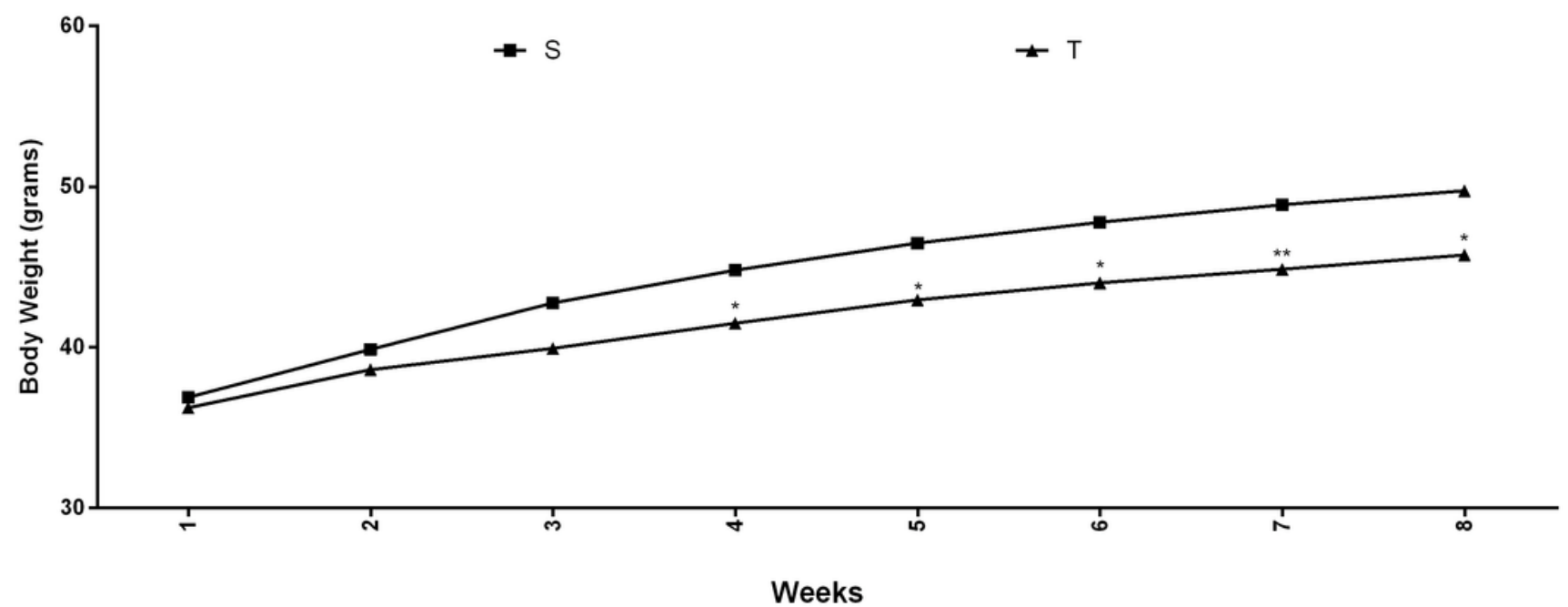

B)

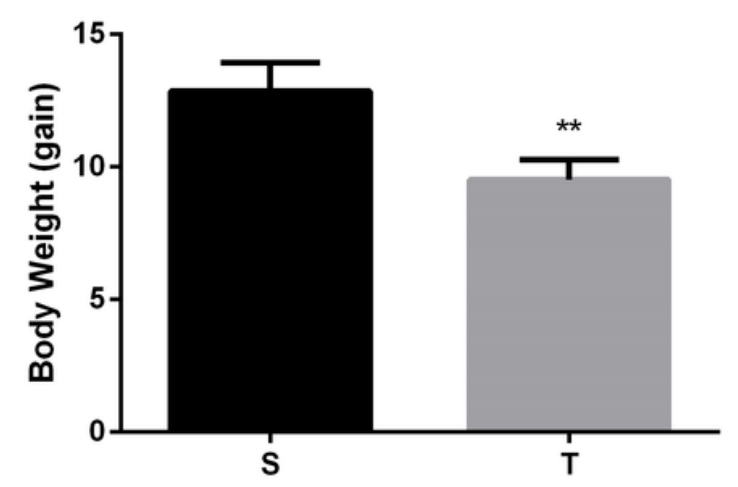

C)

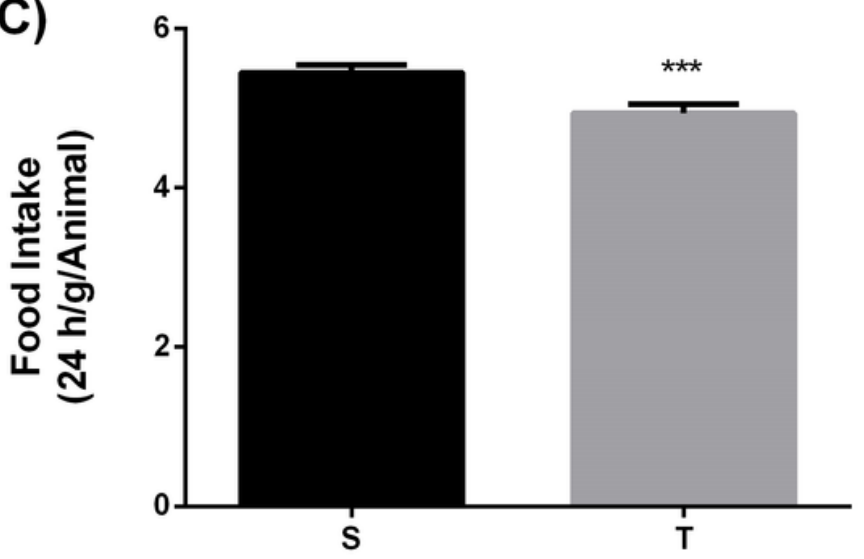

Figure 1

Body index evaluation of ob/ob mice after eight weeks of AET. Analyses of (A) the evolution of body weight by week (in g), (B) body weight (in g), and (C) food intake. ${ }^{*} p<0.05$; ${ }^{* \star} \mathrm{p}<0.01$; ${ }^{* \star *} \mathrm{p}<0.001$. S ( $\left.\mathrm{n}=7\right)$ and $T(n=7)$. 
A)

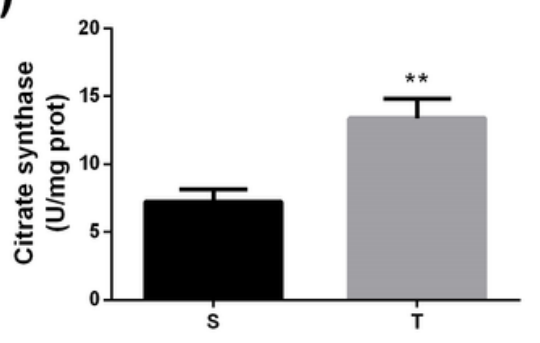

D)

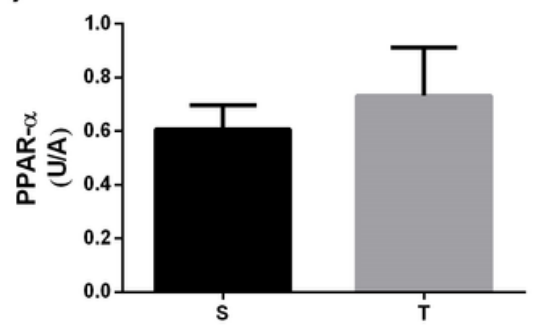

B)

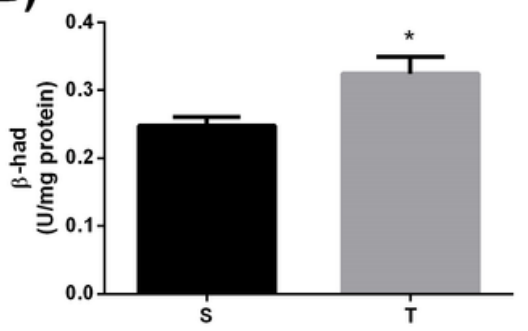

E)

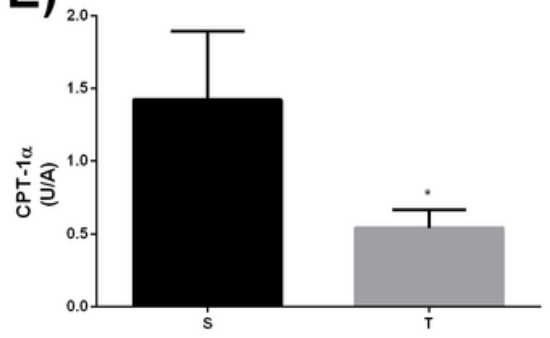

C)

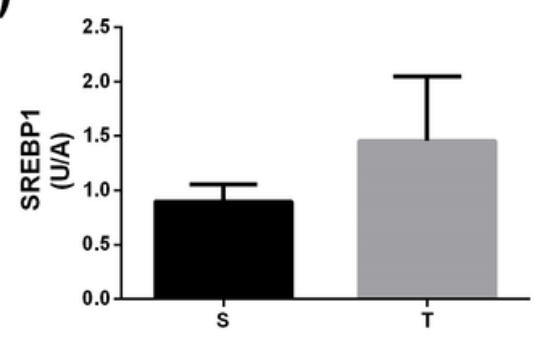

F)

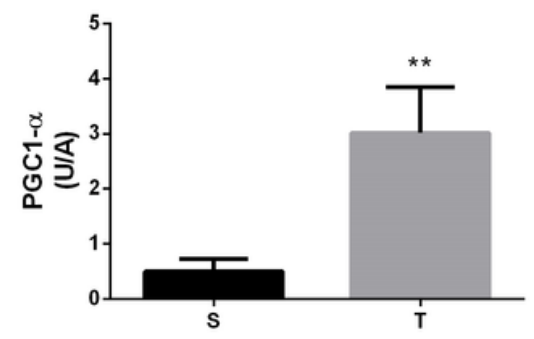

Figure 2

Evaluation of metabolic enzymatic activity (A) citrate synthase activity and (B) $\beta$-hydroxyacetylcodehydrogenase ( $\beta-H A D)$ and the gene expression of $(C)$ sterol regulatory element-binding protein I (SREBP1), (D) peroxisome proliferator-activated receptor alpha (PPAR-】), (E) carnitine palmitoyltransferase I (CPT-10), (F) peroxisome proliferator-activated receptor gamma coactivator 1-alpha (PGC-10) in the liver of ob/ob mice after eight weeks of $A E T$. ${ }^{*} p<0.05 ;{ }^{* \star} p<0.01 ;{ }^{* \star *} p<0.001 ; S(n=6)$ and T $(n=7)$. 
A)

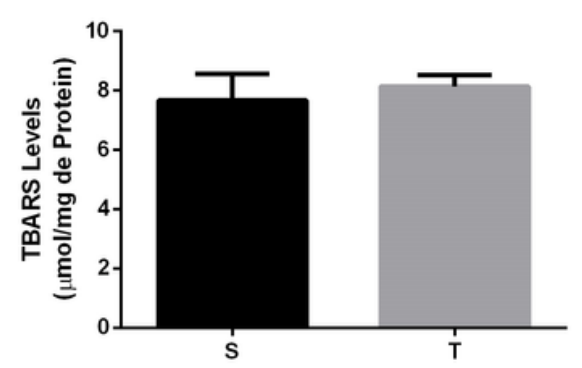

D)

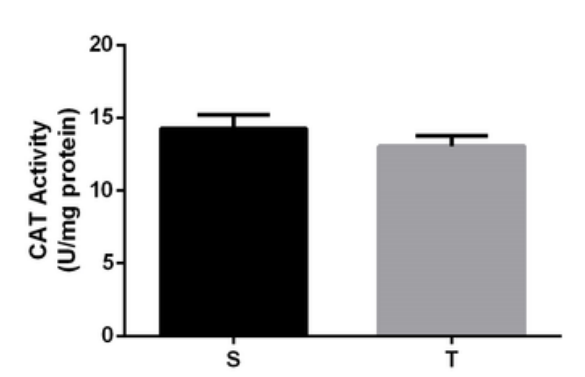

B)

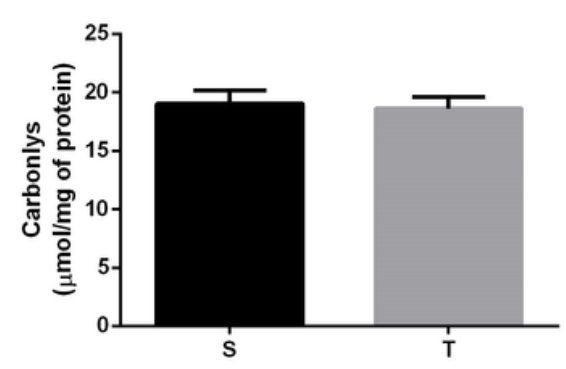

E)
C)

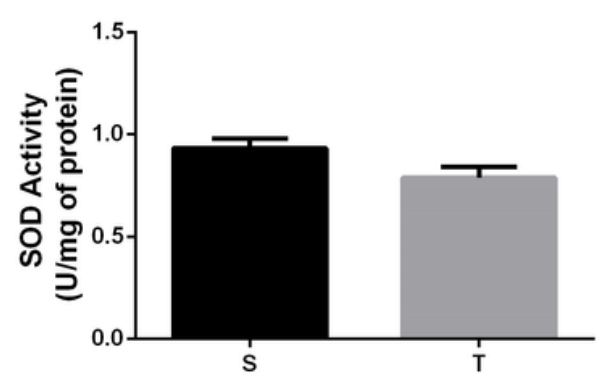

\section{Figure 3}

(A) TBARS and (B) carbonyl levels were evaluated in the liver of ob/ob mice after eight weeks of physical training. Antioxidant enzymatic activity of (C) superoxide dismutase (SOD), (D) catalase (CAT) and (E) glutathione $S$ transferase (GST). ${ }^{*} p<0.05 ; * * p<0.01 ; * * * p<0.001 . S(n=6)$ and $T(n=7)$.

A)

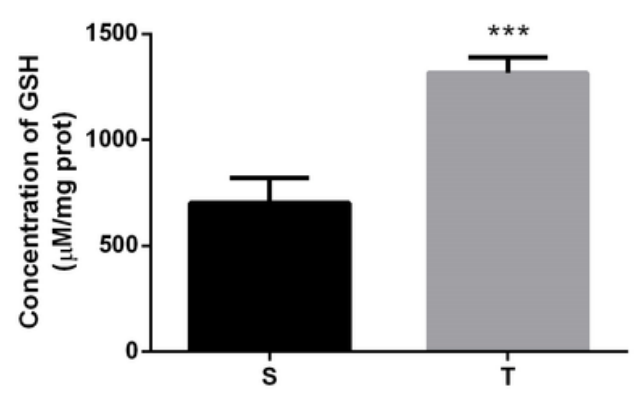

B)

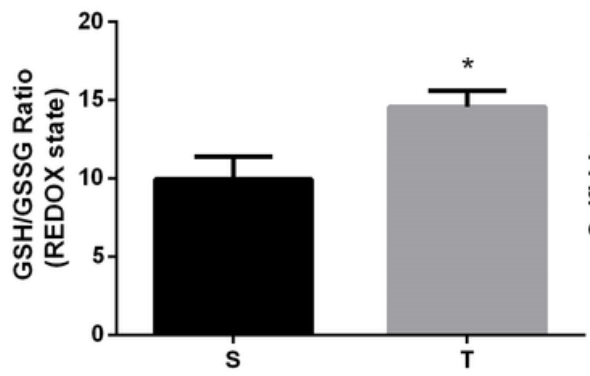

C)

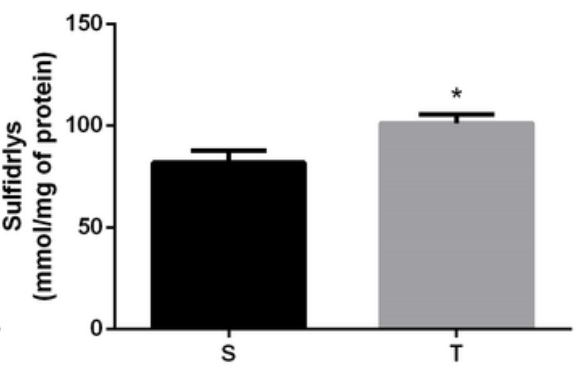

\section{Figure 4}

(A) Reduced glutathione levels (GSH concentration), (B) redox state of glutathione (GSH/GSSG ratio) and (C) total levels of sulfhydryls were evaluated in the liver of ob/ob mice with nonalcoholic fatty liver disease. ${ }^{*} p<0.05 ;{ }^{* \star} p<0.01 ;{ }^{* \star *} p<0.001$. $S(n=6)$ and $T(n=7)$.

\section{Supplementary Files}


This is a list of supplementary files associated with this preprint. Click to download.

- NovoDocumento2019100721.09.53.pdf 